\title{
¿POR QUÉ HAY VERANOS E INVIERNOS? REPRESENTACIONES DE ESTUDIANTES (12-18) Y DE FUTUROS MAESTROS SOBRE ALGUNOS ASPECTOS DEL MODELO SOL-TIERRA
}

\author{
DE MANUEL BARRABÍN, J. \\ IES Joan Miró, Av. Europa, 153, Bellvitge. L'Hospitalet de Llobregat, 08907 Barcelona.
}

\section{SUMMARY}

This paper shows the results of an investigation conducted on more than 900 students aging 12-18, and 50 teacher training students. Misconceptions related with the Sun-Earth model in relation with summers and winters were studied using a single questionnaire and an interview. Changes derived from an instruction based on a simulation activity on the model were also tested, 1,2, and 3 years later. A comparative analysis, between instructed and uninstructed students, also carried out. These analysis and the interpretation of the probable origin of misconceptions are used for some educational suggestions.

\section{INTRODUCCIÓN}

Veranos e inviernos, la diferente duración del día y la noche en las estaciones, los eclipses, las diferencias de horario entre Iugares lejanos del mundo, etc. son hechos y fenómenos que se suceden a lo largo de nuestra vida. Para casi todos ellos tenemos nuestras propias representaciones. De hecho, para gran parte de los conceptos científicos sobre fenómenos y procesos naturales, las personas poseemos nuestras propias ideas, teorías personales (Claxton 1984) que configuran una ciencia intuitiva (Osborne y Freyberg 1985), la cual confiere una cierta funcionalidad, coherencia y capacidad de predicción a nuestro mundo. Estas ideas pueden tener diversos origenes (Pozo et al. 1991) y a menudo no coinciden con los conceptos cientificos vigentes.

En la enseñanza de las ciencias, en el marco del paradigma constructivista, el conocimiento de Ias ideas de los estudiantes sobre conceptos científicos es imprescindible. Se han propuesto diversos modelos de instrucción (Posner et al. 1982, Osborne y Wittrock 1983, Pozo 1989) que parten de las concepciones de los estudiantes, y pretenden reestructurar y cambiar las ideas alternativas.
En diferentes momentos del currículo de la enseñanza primaria son tratados diversos conceptos de cosmología, entre los que se incluye el modelo Sol-Tierra. Frecuentemente estos contenidos no vuelven a ser considerados de un modo directo en la enseñanza secundaria. No obstante, algunos temas relacionados con la astronomía aparecen con intermitencia en la ciencia escolar, y quizás con más frecuencia en los aprendizajes no formales de los adolescentes. El aprendizaje de un modelo adecuado Sol-Tierra facilita la comprensión de fenómenos como: la sucesión estacional a lo largo del año, el sistema horario, la diferente duración de los días y de las noches en el ciclo anual y, en general, de una serie de situaciones derivadas de fenómenos naturales que a menudo no tienen una explicación sencilla ni deducible a partir de la lógica y del sentido común.

Se han realizado numerosas investigaciones para conocer las concepciones asociadas al modelo cosmológico Sol-Tierra. Las más relevantes, con su metodología y resultados, se encuentran esquematizadas en la tabla l. 
Tabla I

Cuadro esquemático de las investigaciones sobre las concepciones del modelo Sol-Tierra.

\begin{tabular}{|c|c|c|c|}
\hline Referencia & Muestra y metodología & Conceptos investigados & Concepciones más relevantes detectadas \\
\hline Giordan y de Vecchi (1987) & $\begin{array}{l}76 \text { niños (9-11 años) } \\
\text { Cuestionario y } \\
\text { entrevișta }\end{array}$ & Modelo heliocéntrico & $\begin{array}{l}\text { - Visión heliocéntrica (correcta) } 80 \% \text {. Aunque en las } \\
\text { cntrevistas muchos muestran una visión geocénttica }\end{array}$ \\
\hline $\begin{array}{l}\text { Jones, Lynch y Reesinch } \\
(1987)\end{array}$ & $\begin{array}{l}32 \text { niños (9-12 años) } \\
\text { Entrevista }\end{array}$ & $\begin{array}{l}\text { Representaciones } \\
\text { Tierra-Sol-Luna: } \\
\text { forma, taman̄o } \\
\text { movimientos }\end{array}$ & $\begin{array}{l}\text { - } 5 \text { modelos espaciales del sistema Sol-Tierra-Luna } \\
\text { ( } 3 \text { geocéntricos y } 2 \text { heliocéntricos) } \\
\text { - Formas bidimensionales de estos astros } \\
\text { - Formas tridimensionales (no esféricas) } \\
\text { - Formas esfëricas } \\
\text { - Sol, Tierra y Luna del mismo tamaño } \\
\text { - Dos astros del mismo tamaño y uno diferente }\end{array}$ \\
\hline Kapterer y Dubois (1981) & $\begin{array}{l}\text { Niños, adolescentes y } \\
\text { adultos } \\
\text { Cuestionario }\end{array}$ & Modelo heliocéntrico & - Visión geocéntrica, $30,5 \%$ \\
\hline Klein (1982) & $\begin{array}{l}24 \text { niños ( } 7-8 \text { años) } \\
\text { Entrevista }\end{array}$ & $\begin{array}{l}\text { Sistema Sol-Tierra } \\
\text { Forma y tamaño } \\
\text { Movimiento de rotación } \\
\text { y dias y noches }\end{array}$ & $\begin{array}{l}\text { - La Tierra es más grande que el Sol } \\
\text { - La Tierra y el Sol tienen el mismo tamaño } \\
\text { - Dias y noches no se deben a la rotación sino a que el } \\
\text { Sol se "esconde" (cae verticalmente) }\end{array}$ \\
\hline Nussbaum y Novak (1976) & $\begin{array}{l}60 \text { nin̄os ( } 8-9 \text { años) } \\
\text { Lintrevista }\end{array}$ & $\begin{array}{l}\text { La Tierra como cuerpo } \\
\text { cósmico. Forma } \\
\text { Dirección de caída de } \\
\text { los objetos }\end{array}$ & $\begin{array}{l}\text { - La Tierra es plana, no es esférica } \\
\text { - La Tierra es una bola compucsta por dos hemisferios, } \\
\text { el inferiot es sólido, el superior es el cielo (airc) } \\
\text { - Concepto de atriba y abajo en el espacio cósmico. } \\
\text { Objetos en el hemisfetio sur «caen hacia el cielo». } \\
\text { - La Tierra es esférica pero no hay relación arriba-abajo } \\
\text { respecto al centro de la Tierra }\end{array}$ \\
\hline Schoon (1992) & $\begin{array}{l}1.213 \text { estudiantes } \\
\text { (ensenanza elemental, } \\
\text { secundaria y adultos) } \\
\text { Cuestionario }\end{array}$ & $\begin{array}{l}\text { Diversas representa- } \\
\text { ciones y conceptos } \\
\text { de la Tierra y dei } \\
\text { espacio }\end{array}$ & $\begin{array}{l}\text { - A las } 12 \text { det mediodía el Sol está verticalmente por } \\
\text { encima nuestro, } 82,4 \% \\
\text { - En verano hace más calor que en invierno porque la } \\
\text { Tierra está más cerca del Sol, } 77,6 \% \\
\text { - En mayo, junio y julio el Sol se pone por el oeste, } 58 \% \\
\text { - En cada dia de verano la cantidad de luz diuma es } \\
\text { mayor que la dei dia anterior, } 32,4 \% \\
\text { - La Luna tarda un año en dar la vuelta a la Tierra, } \\
19,5 \% \\
\text { - La Luna brilla porque es como una gran estrella } 15,7 \% \\
\text { - Días y noches se dan al ir la Tjersa alrededor del Sol, } \\
\text { 19,6\% } \\
\text { - Días y noches se dan porque el Sol va alrededor de la } \\
\text { Tierra (visión geocéntrica), } 8,8 \%\end{array}$ \\
\hline
\end{tabular}

Este trabajo tiene como propósito la detección de representaciones que tienen estudiantes de 12 a 18 años y futuros maestros sobre algunos aspectos del modelo SolTierra, especialmente sobre el modelo de órbita terrestre y en las causas que hacen que haya veranos e inviernos. Pretende también explicitar ciertas implícaciones didâcticas a partir de las concepciones encontradas y de sus posibles orígenes. Finalmente, se investigan los mismos conceptos uno, dos y tres años después de realizar una determinada instrucción, basada en una simulación son bre dicho modelo.

\section{DISEÑO EXPERIMENTAL}

Se preparó tun cuestionario (Fig. 1) para detectar las ideas de los alumnos con relación a algunos aspectos del modelo. En una primera pregunta se debía escoger entre 3 dibujos correspondientes a diferentes modelos de órbita terrestre (A, trayectoria elíptica con ia Tierra situada en uno de los focos; B, trayectoria elíptica con la Tierra situada en el centro; C, trayectoria circular con la Tierra en el centro). Posteriormente se pedía la aplicación de algunas de las bases teóricas del modelo; primero en una 
pregunta abierta (Da una explicación de por qué en verano hace calor-hace más sol-y en invierno hace frio - hace menos sol-) y, en segundo lugar, en una situación problema (En Australia -hemisferio sur- mucha gente celebra la Navidad bañándose en la playa. Explica por qué en el hemisferio sur es verano cuando en el hemisferio norte-nosotros-es invierno). También partiendo de dicho cuestionario se diseñó un modelo de entrevista con el propósito de profundizar en las concepciones de los estudiantes.

\section{Figura 1}

Cuestionario usado en esta investigación.

Como ya sabes, Ia Tierra gira alrededor del Sol mediante un movimiento denominado traslación. Tarda 1 año en dar una vuelta completa. A lo largo del año, nosotros tenemos distintas estaciones que se suceden: verano, otono, invierno y primavera.

1. ¿Cuál dée estos tres dibujos se aproxima más a la trayectoria que sigue la Tierra alrededor del Sol? (Pon un círculo en la letra).

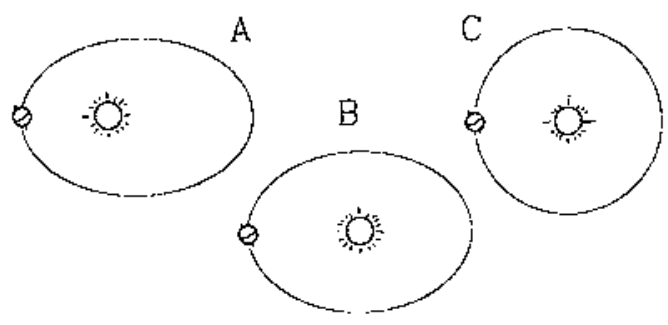

2. Da una explicación de por qué en verano hace calor y en invierno hace frío.

3. En Australia (hemisferio sur) mucha gente celcbra las Navidades bañándose en la playa. Explica por qué en el hemisferio sur es verano cuando en el hemisferio norte (nosotros) es invierno.

El cuestionario fue realizado por 904 estudiantes (en una proporción genérica aproximada del $50 \%$ ), pertenecientes a 11 centros de enseñanza primaria y secundaria de Cataluña. La muestra se estratificaba en la edad (12 a 18 años), en el currículo (primaria, formación profesional, nueva secundaria obligatoria y bachillerato) y en el marco socioeconómico del centro ( 6 centros públicos y 5 privados; 5 en áreas urbanas, 4 en áreas suburbanas, 2 en áreas rurales relativamente urbanizadas). También realizaron el cuestionario 50 estudiantes de magisterio (de $1^{2}$ y $2^{\circ}$ curso de 2 escuelas universitarias de formación de profesorado). Las características de las poblaciones investigadas se muestran en la tabla II.

También se llevaron a cabo entrevistas partiendo de las situaciones problema planteadas en el cuestionario. Para ello se eligieron diversos alumnos al azar que previamente habían realizado el cuestionario.
La corrección de los cuestionarios fue realizado mediante el establecimiento de categorías de respuestas para facilitar el análisis de los resultados. Las entrevistas fueron transcritas y las respuestas contribuyeron al establecimiento de las categorías y a la detección de concepciones.

En el 1r. curso de bachillerato de dos de Ios centros de secundaria, se había realizado en el primer trimestre de curso, dentro de los contenidos de geología, una actividad en relación con las estaciones y la duración de días y noches. La actividad, de unos 50 minutos de duración consistía en una simulación en una aula oscura, partiendo de superficies planas que se incinaban, en las cuales incidía un haz iluminado (diapositiva recortada), pasando posteriormente a un modelo más complejo con un balón (Tierra) y el foco de luz (SoI), donde se introducían variables como la inclinación del eje de la Tierra o la distancia al Sol (para más detalles ver de Manuel y Montero 1992). Con el mismo cuestionario se investigaron las concepciones del modelo en diferentes grupos que hacían uno, dos y tres años, respectivamente, que habían realizado la simulación, con el fin de evaluar si este tipo de instrucción había modificado las concepciones más comunes. Los resultados de esta muestra no se tuvieron en cuenta a la hora de realizar el cómputo global por edades, $y$ fueron comparadas posteriormente con el cómputo conjunto de otros grupos del mismo nivel educativo (es decir, $2^{\circ}$ y $3^{\circ}$ de BUP y COU) que no habían realizado la simulación.

\section{Tabla II}

Características de la muestra de estudiantes que realizaron el cuestionario de esta investigación (Fig. 1).

\begin{tabular}{|c|c|}
\hline Edad & Muestra \\
\hline $12-13$ & $\begin{array}{l}5 \text { grupos-clase }\left(4 \text { de } 7^{2} \text { de EGB, } 1 \text { de } 1^{\circ} \text { de ESO) }\right. \\
4 \text { centros } \\
n=128\end{array}$ \\
\hline $13-14$ & $\begin{array}{l}5 \text { grupos-clase ( } 4 \text { de } 8^{\circ} \text { de EGB, } 1 \text { de } 2^{9} \text { de ESO) } \\
4 \text { centros } \\
\mathrm{n}=116\end{array}$ \\
\hline $14-15$ & $\begin{array}{l}8 \text { grupos-clase }\left(5 \text { de } 1^{5} \text { de BUP, } 3 \text { de } I^{9} \text { de FP) }\right. \\
6 \text { centros } \\
\mathrm{n}=209\end{array}$ \\
\hline $15-16$ & $\begin{array}{l}8 \text { grupos-clase }\left(7 \text { de } 2^{9} \text { de BUP, } 1 \text { de } 2^{2} \text { de FP) }\right. \\
4 \text { centros } \\
n=230\end{array}$ \\
\hline $16-18$ & $\begin{array}{l}10 \text { grupos-clase ( } 7 \text { de } 3^{I} \text { de BUP, } 3 \text { de COU) } \\
7 \text { centros } \\
n=221\end{array}$ \\
\hline$>18$ & $\begin{array}{l}2 \text { grupos clase }\left(1^{9} \text { de magisterio, especialidad }\right. \\
\text { lengua cxtranjera; } 2^{2} \text { de magisterio, especialidad } \\
\text { ciencias) } \\
2 \text { centros } \\
\mathrm{n}=50\end{array}$ \\
\hline
\end{tabular}




\section{RESULTADOS}

En la figura 2 pueden observarse los porcentajes de respuestas para cada grupo de edad en relación con el modelo de órbita terrestre (cuestión 1). La elección de trayectorias elípticas (A y B) fue Ia más frecuente. $\mathrm{La}$ trayectoria circular (C) fue minoritaria, y elegida por muy pocos chicos y chicas de 15 a 18 años. La elección de la trayectoria elíptica con la Tierra en el centro (B) fue mayoritaria en estudiantes de 13 a 14 años, al contrario que en los otros grupos de edad estudiados.

\section{Figura 2}

Representación de los porcentajes por edades referidos a las respuestas dadas en la pregunta 1 (Fig.1, modelo orbital): A, orbita elíptica con el Sol aproximadamente en uno de los focos; B, órbita eliptica con el Sol en el centro; C, órbita circular con el Sol en el centro.

Los asteriscos corresponden a los porcentajes de las respuestas dadas por los estudiantes de magisterio.

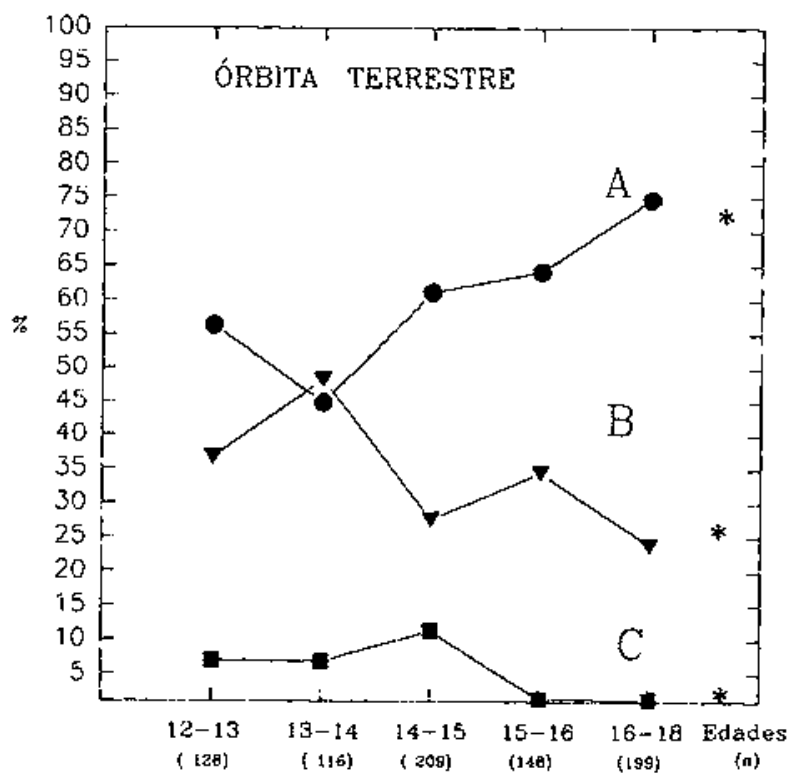

Pocos estudiantes dieron una explicación satisfactoria al porqué de veranos e inviernos. La concepción más común fue explicarlo por la distancia a la que la Tierra se encuentra del Sol en su trayectoria alrededor de éste (verano, más cerca; invierno, más lejos) (Fig. 3). Concepción muy persistente, que se dio con una frecuencia semejante en las distintos grupos de edad estudiados (así como en un $60 \%$ de estudiantes de magisterio). En esta concepción se distinguieron dos categorías: aquellos estudiantes que explicaron el calor o el frío únicamente por la distancia aI Sol, y aquéllos que además mencionaron Ia inclinación del eje de rotación terrestre (Fig. 4).

En la tabla III se muestran los porcentajes, por edades, de las principales ideas alternativas detectadas en las respuestas de los cuestionarios; en la figura 6 se exponen dibujos, realizados por los estudiantes, que ilustran algunas de las concepciones aparecidas.

Figura 3

Representación de los porcentajes por edades referidos a las respuestas dadas en la pregunta 2 (Fig.1, ${ }_{b}$ Por qué en verano hace calor y en invierno hace frio?). En la tabla III se muestran otras categorías de respuestas dadas en dicha pregunta.

Los asteriscos corresponden a los porcentajes de las respuestas dadas por los estudiantes de magisterio.

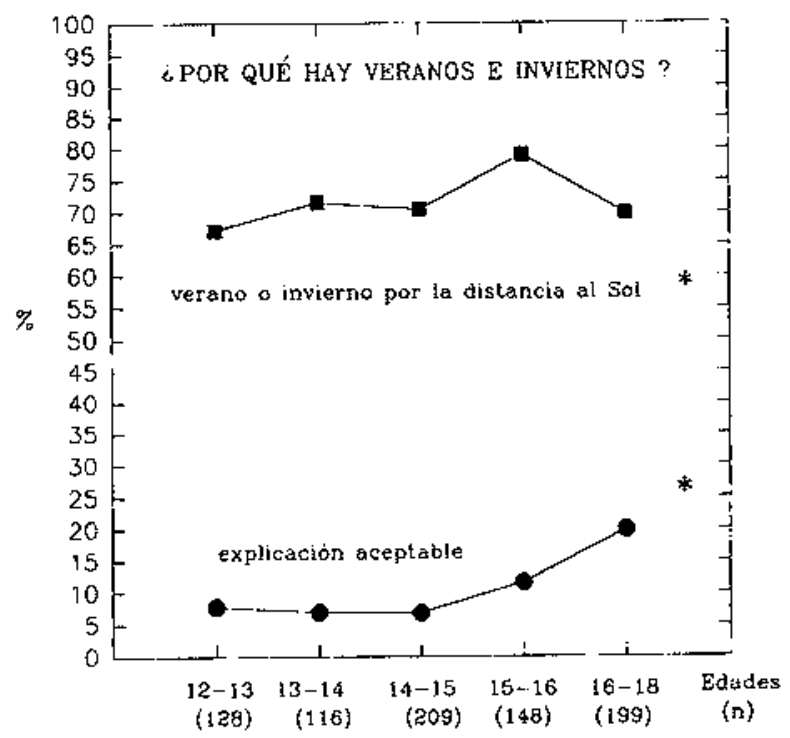

Figura 4

Representación de los porcentajes por edades de la concepción «verano o invierno por la distancia al Sol» (Fig. 3). Se scparan los porcentajes de respuestas en las que se da la distancia como única causa, de las respuestas que además hacen referencia a la inclinación del eje terrestre.

Los asteriscos corresponden a los porcentajes de las respuestas dadas por los estudiantes de magisterio.

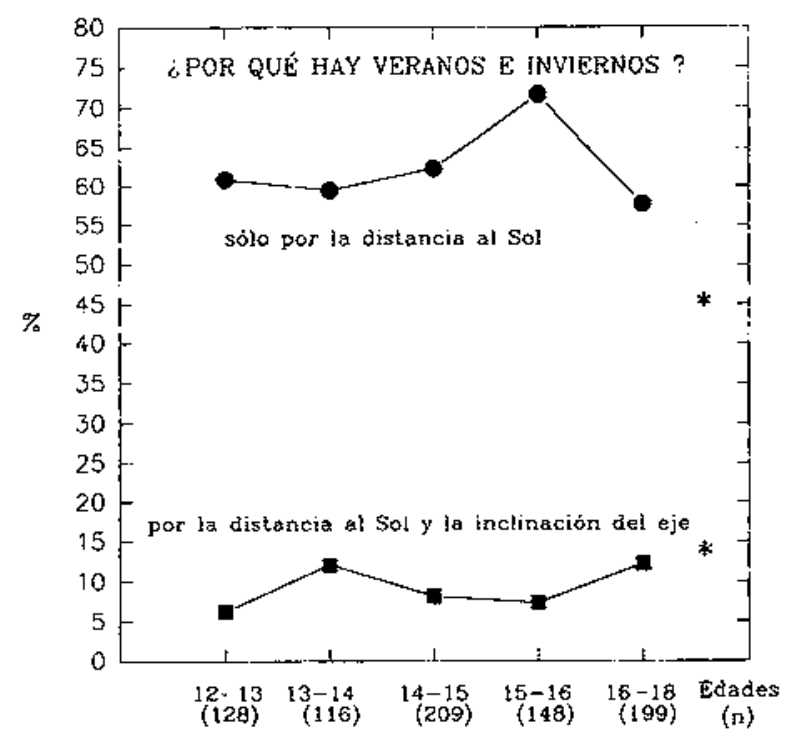


Tabla III

Categorias de respuestas dadas en las preguntas 2 y 3 del cuestionario. Los resultados se expresan en tantos por ciento, sin incluir en el cómputo aquellos grupos-clase que recibieron la instrucción que se comenta en el texto. Se entiende por «respuesta aceptable» todas aquéllas que hicieron referencia a la inclinación del eje de la Tierra 0 al grado de incidencia de los rayos solares para justificar la situación de verano o invierno.

\begin{tabular}{|c|c|c|c|c|c|c|}
\hline & $\begin{array}{c}12-13 \\
n=128\end{array}$ & $\begin{array}{c}13-14 \\
n=116\end{array}$ & $\begin{array}{c}14 \cdot 15 \\
n=208\end{array}$ & $\begin{array}{c}15-16 \\
n=148\end{array}$ & $\begin{array}{c}16 \cdot 18 \\
n=155\end{array}$ & $\begin{array}{l}\text { Estud. magist. } \\
\qquad \mathbf{n}=\mathbf{5 0}\end{array}$ \\
\hline \multicolumn{7}{|l|}{ 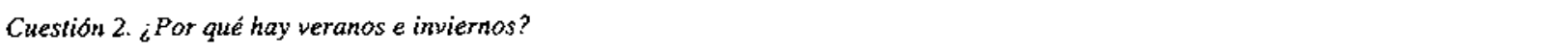 } \\
\hline Respuesta aceptable & 7,8 & 6,9 & 6,8 & 11,5 & 19,9 & 22 \\
\hline Se debe a la distancia de la Tierra al Sol & 60,9 & 59,5 & 62,3 & 71,6 & 57,7 & 46 \\
\hline Distancia más inclinación del eje terrestre & 6,3 & 12,1 & 8,2 & 7,4 & 12,2 & 14 \\
\hline Se debe al movimiento de rotación de la Tierra & 11,7 & 9,5 & 6,3 & 4,1 & 4,5 & 8 \\
\hline Representación bidimensional & 4,7 & 1,7 & 3,8 & 0,7 & 2,5 & 6 \\
\hline Otras respuestas & 8,6 & 10,3 & 12,6 & 4,7 & 3,2 & 4 \\
\hline \multicolumn{7}{|l|}{ Cuestion 3. Aplicacion del modelo } \\
\hline Respuesta aceptable & 12 & 12,4 & 17,7 & 29,5 & 34,9 & 32 \\
\hline Más cerca al Sol por la inclinación del eje & 10,4 & 14,2 & 9,4 & 11,6 & 14,5 & 30 \\
\hline Más cerca al Sol (hemisferio sur en Navidad) & 9,6 & 3,5 & 9,9 & 1,4 & 5,9 & 4 \\
\hline Se debe al movimiento de rotación de la Tierra & 20,8 & 17,7 & 24,6 & 19,9 & 17,1 & 20 \\
\hline El sur es siempre más cálido & 10,4 & 9,7 & 5,4 & 6,8 & 1,3 & 4 \\
\hline Visiön bidimensional & 8,8 & 5,3 & 6,4 & 9,6 & 9,2 & 4 \\
\hline Aquí verano, allí invierno & 14,4 & 17,7 & 11,3 & 13 & 7,2 & 4 \\
\hline Otras respuestas & 13,6 & 19,7 & 15,3 & 8,2 & 9,9 & 2 \\
\hline
\end{tabular}

El hecho de que muchos estudiantes justificasen veranos e inviernos por la rotación terrestre demuestra las dificuitades propias del modelo. En este sentido, una representación interesante detectada en las dos últimas cuestiones fue entender el modelo Sol-Tierra en un plano, no en el espacio. Esta representación les permite explicar mejor su modelo de que las estaciones dependen de la distancia entre el Sol y la Tierra, y que hay zonas de ésta permamentemente sin radiación, lo cual es el argumento de muchos estudiantes para justificar el invierno (ipero no la noche!) (Fig. 6, 10-13). El điseño del cuestionario no permitió detectar claramente esta concepción; sin embargo, ésta pudo identificarse cuando se realizaba un dibujo o en las entrevistas. Por ello, posiblemente esta representación se presente realmente con más frecuencia de la que se muestra en la tabla III.

En la situación problema (cuestión 3), al aplicar el modelo, se observaron (Fig. 5) resultados más favorables que en la cuestión anterior (Figs. 3 y 4). Abundaron más las respuestas haciendo referencia a la inclinación del eje terrestre y al grado de incidencia de los rayos solares. Aparecieron, no obstante, nuevas concepciones (en el sur siempre hace más calor), se confirmaron otras ya aparecidas (se debe a la rotación, el eje inclinado hace que el sur estế más cerca đel Sol, representación biđimensional del modelo) y se dieron respuestas teleológicas («cuando aquí es invierno, allí tiene que ser verano», «el mundo está hecho así», etc.).

\section{Figura 5}

Representación de los porcentajes por edades en relación con las respuestas a la pregunta 3 (Fig.1). En la tabla II se muestran otras categotias de respuestas dadas en dicha pregunta. Los asteriscos corresponden a los porcentajes de las respuestas dadas por los estudiantes de magistcrio.

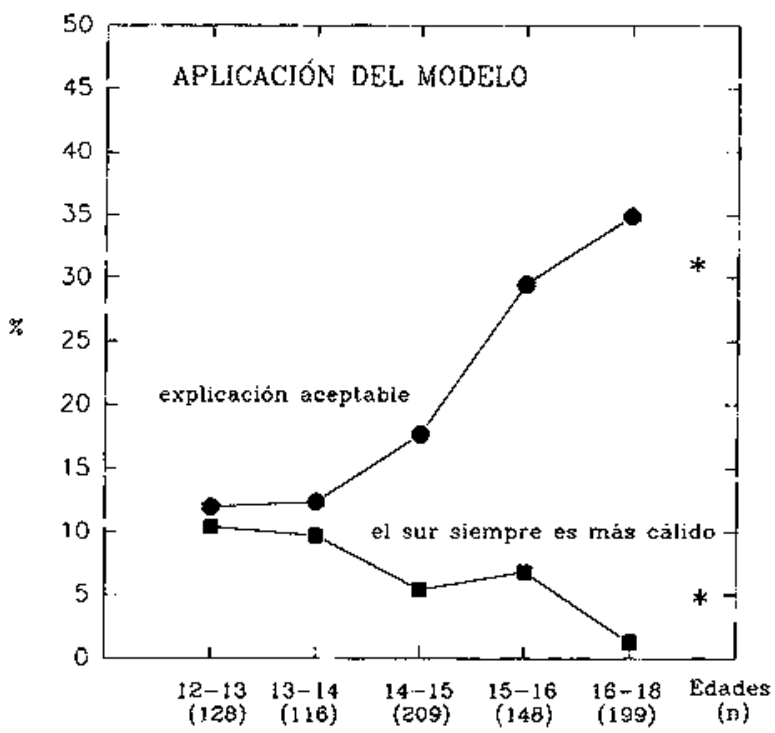


Figura 6

Dibujos seleccionados (procedentes de cuestionarios y entrevistas) que ilustran algunas de las concepciones detectadas.
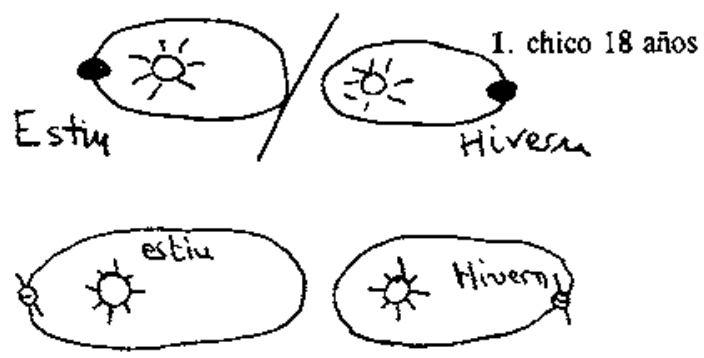

3. chico 13 años

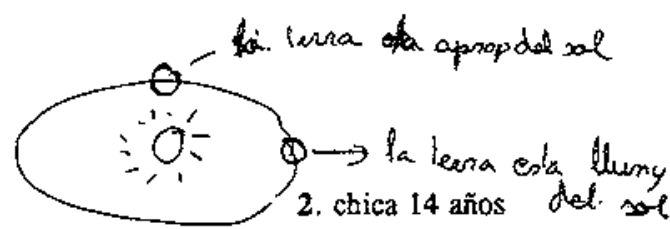

- texaltucs (Ap calex)

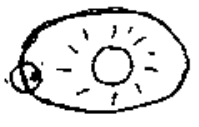

- inosattien

(is the

4. chica 14 años

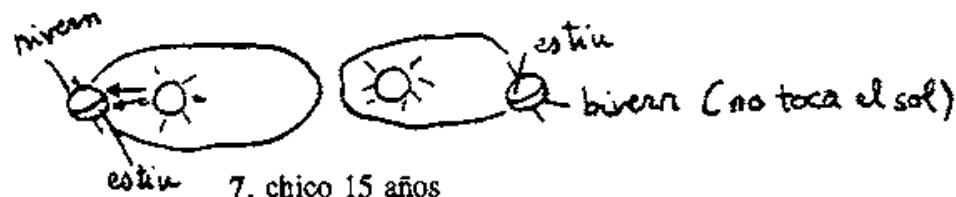

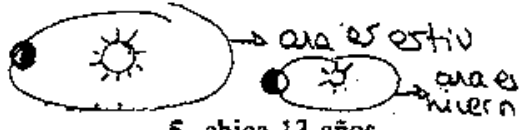

5. chica 13 aก̃os

estive 7. chico 15 años

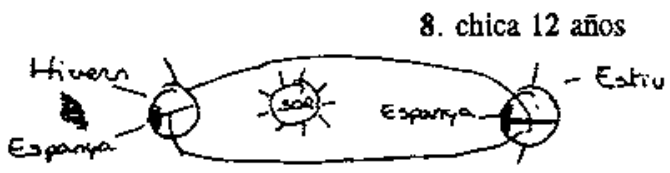

teric

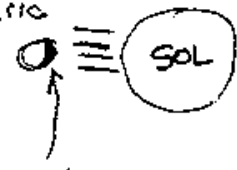

calor

6. chico 13 años

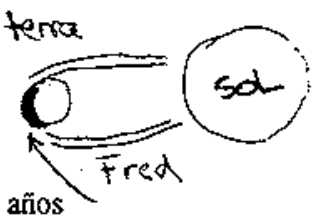

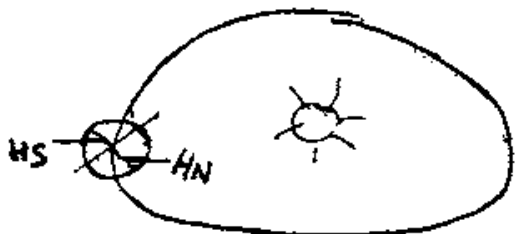

9. chica 19 años
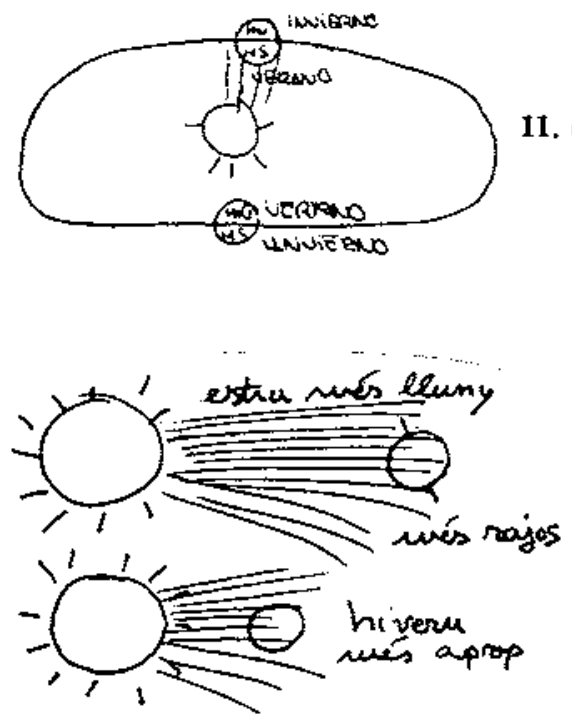

14. chico 17 años

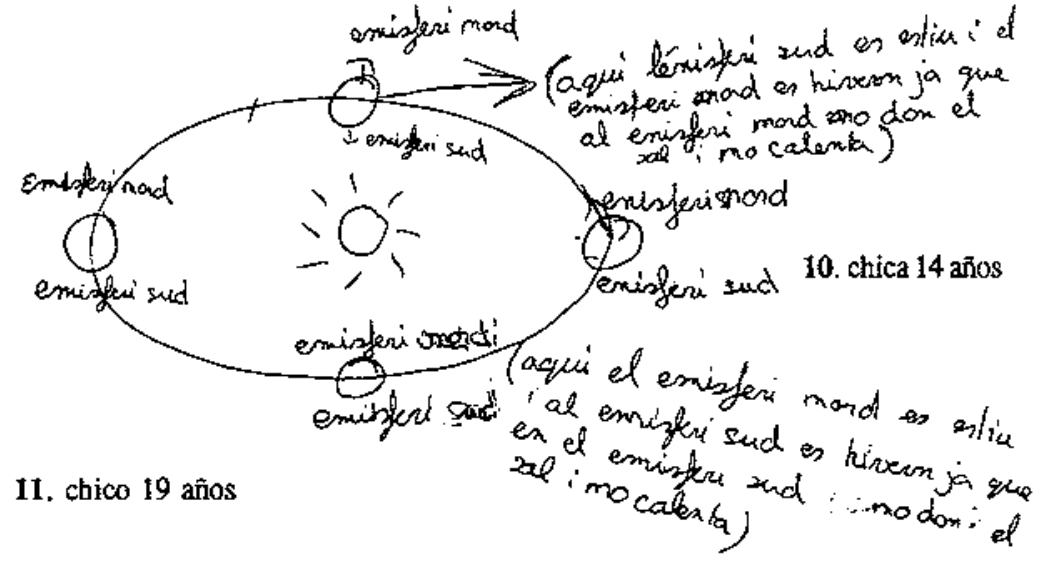

Perque dona el sò

12. chica 13 años

Perque cona soil al nord.

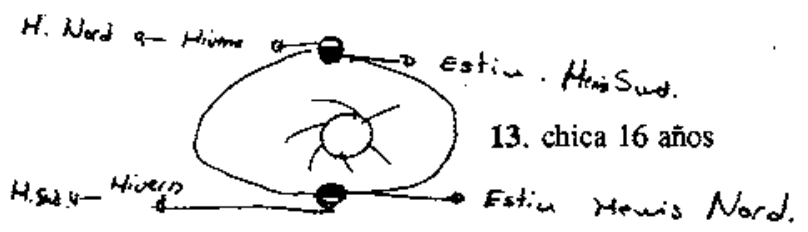


La visión geocéntrica se encontró en tres casos, a pesar de que los dibujos y el texto introductorio del cuestionario explicitaban la visión heliocéntrica.

En Ia tabla IV y en la figura 7 se comparan los porcentajes de respuestas aceptables dadas globalmente por los grupos estudiados (control) con las respuestas dadas por grupos de estudiantes que realizaron Ia actividad de simulación uno, dos o tres años atrás. Hay diferencias significativas entre la respuestas dadas por los grupos control y las de aquellos grupos que habían realizado la simulación. Las diferencias son más importantes en los alumnos mayores (aquéllos que realizaron la actividad tres cursos atrás).

\section{DISCUSIÓN}

\section{Origen de las concepciones}

Asumiendo que la Tierra, en su trayectoria alrededor del Sol, no está siempre a la misma distancia de éste, que en verano haga calor por estar más próximos al Sol y en invierno haga frío (menos calor) por estar más lejos de éste, es una concepción espontánea analógica (Pozo et al. 1991). Es una representación que nace del sentido común y de la lógica, muy consistente con independencia de la edad (Fig. 3). También es una concepción común, con una frecuencia superior al $75 \%$ (Tabla I), que Schoon (1992) detectó en una investigación realizada en más de 1.200 estudiantes de diversas edades. Camino (1995), en una reciente investigación sobre las ideas de estudiantes de magisterio, también detecta esta común representación $(35 \%)$.

Explicar veranos e inviernos por la rotación terrestre (Fig. 6, 4-6) fue otra concepción importante: «la Tierra está mirando al Sol en verano, y está de espaldas al Sol en invierno» (chico de 12 años); «la Tierra va dando vueltas sobre sí misma, por tanto cuando en un lugar es invierno en el otro hemisferio es verano» (chica de 13 años). Aunque algunas veces no fue posible diferenciar esta concepción de la tepresentación en un plano del modelo Sol-Tierra (Fig. 6, 7-8): «Ia Tierra va girando y, si nos hallamos en el lugar contrario del Sol, no nos dará tanto" (chico de 13 años); "la Tierra gira sobre su eje, y por eso hay determinadas épocas en que un hemisferio está más cerca del Sol que otro" (chica de 17 años).

La concepción compartida por algunos estudiantes de que en el hemisferio sur hace siempre más calor que en el norte, probablemente sea debida a que extrapolan situaciones regionales a una escala planetaria: «en el hemisferio sur no hace frío ni nieva. En cambio en el hemisferio norte nieva» (chico de 13 años); «en la parte sur siempre da más luz del Sol, y por esto siempre hace calor» (chica de 14 años); «al encontrarse en el hemisfe-

Tabla IV

Comparación de resultados, expresados en tantos por ciento, entre las tespuestas dadas al cuestionario por estudiantes de 15 -16 con 2 grupos clase que habían realizado una actividad de simulación del modelo hace un año. Y entre los resultados de la muestra 16-18 con dos grupos que realizaron hace dos y tres años respccivamente una actividad de simulación del modelo.

\begin{tabular}{|c|c|c|c|c|c|}
\hline & $\begin{array}{l}15-16 \\
n=148\end{array}$ & $\begin{array}{l}15-16 \\
1 \text { año } \\
n=82\end{array}$ & $\begin{array}{c}16-18 \\
n=155\end{array}$ & $\begin{array}{l}16-17 \\
2 \text { años } \\
n=55\end{array}$ & $\begin{array}{l}17-18 \\
3 \text { años } \\
n=22\end{array}$ \\
\hline \multicolumn{6}{|l|}{ Cuestión 1} \\
\hline Opción A (órbita elíptica, Sol en un foco) & 64,2 & 81,7 & 74,8 & 86,7 & 100 \\
\hline Opción B (6́rbita elíptica, Sol en cI centro) & 34,5 & 15,9 & 23,9 & 10 & 0 \\
\hline Opción C (órbita circular, Sol en el centro) & 1,4 & 2,4 & 1,3 & 3,3 & 0 \\
\hline \multicolumn{6}{|l|}{ 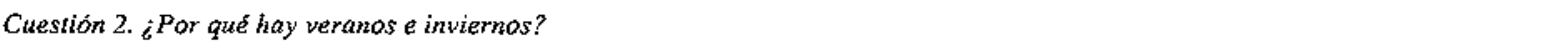 } \\
\hline Respuesta aceptable & 11,5 & 17,1 & 19,9 & 16,7 & 40,9 \\
\hline Se debe a la distancia de la Tierra al Sol & 71,6 & 54,9 & 57,7 & 70 & 50 \\
\hline Distancia más inclinación del eje terrestre & 7,4 & 14,6 & 12,2 & 6,7 & 4,5 \\
\hline Se debe al movimiento de rotación de la Tierra & 4,1 & 3,7 & 4,5 & 3,3 & 4,5 \\
\hline \multicolumn{6}{|l|}{ Cuestión 3. Aplicación del modelo } \\
\hline Respuesta accptable & 29,5 & 39 & 34,9 & 36,7 & 45,5 \\
\hline Más cerca al Sol por la inclinacjón del eje & 11,6 & 11 & 14,5 & 6,7 & 13,6 \\
\hline Más cerca al Sol (hemisferio sur en Navidad) & 1,4 & 2,4 & 5,9 & 3,3 & 0 \\
\hline Se debe al movimiento de rotación de la Tierra & 19,9 & 22 & 17,1 & 30 & 27,3 \\
\hline El sur es siempre más cálido & 6,8 & 3,7 & 1,3 & 13,3 & 4,5 \\
\hline
\end{tabular}


Eigura 7

Comparación entre las respuestas de estudiantes que realizaton una actividad de simulación del modelo Sol-Tierra en el primer curso de bachillerato (hace 1, 2 o 3 años: columnas de la derecha), y las respuestas de estudiantes del mismo nivel educativo que no realizaron dicha actividad (control: columnas de la izquierda).

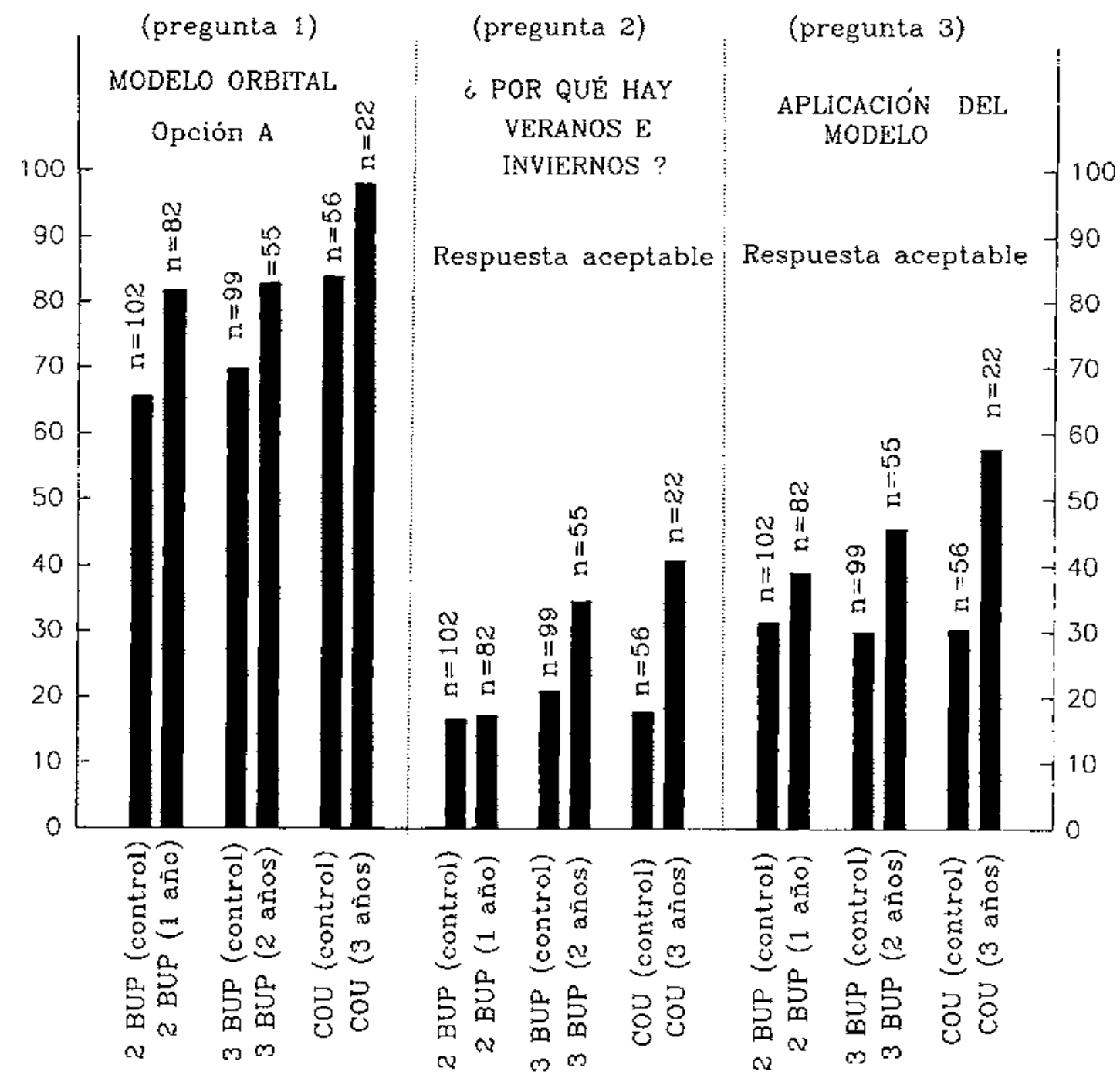

rio sur recibe durante todo el año con más fuerza los rayos đel Sol» (chico de 17 años); «el hemisferio sur está situado por debajo del Ecuador, de manera que para ellos siempre es verano» (chica de 15 años); «el Sol siempre está más cerca del hemisferio sur, esté la Tierra en la posición que esté, en el hemisferio sur siempre hace más calor» (chico de 17 años). Algunos estudiantes opinaron que en el sur hace más calor porque el Sol siempre está más cerca del sur que del norte, creencia que pudo ser inducida por la imagen del eje inclinado (Fig. 1).

Otras interpretaciones del modelo, interesantes pero minoritarias, fueron la relación de las estaciones con la velocidad de rotación terrestre (concepción probablemente creada por un pensamiento causal, ya que las horas de luz diaria cambian con las estaciones), o con el tiempo que tarda la luz en llegar a la Tierra. Las presentaron algunos estudiantes de distintas edades: «en verano la Tierra gira más lentamente, y por eso los días són más largos» (chica de 14 años); «en verano la rotación es más lenta, por esto hay más horas de Sol" (chica de 17 años); «los rayos del Sol tardan más en llegar al estar más lejos, por eso es invierno» (chico de 17 años); «es verano porque estamos más encarados al Sol, y la Tierra gira más poco a pocon (chico de 17 años).

Una representación más compleja que la simple analogía mostrada por la mayoría fue la idea de que la luz del Sol îlumina más la Tierra si ésta se encuentra lejos que si se balla cerca (Fig. 6, 14): «en verano la Tierra tiene más 
Sol porque está más lejos, y en invierno está más cerca y coge menos calor» (chica 14 años); o «al alejarse la Tierra del Sol, tiene más horas de luz. Por eso hace más calor» (chico de 16 años).

Muchos estudiantes probablemente habian oido hablar o habían leído (en el contexto escolar o fuera de él) sobre la importancia de la inclinación con la que incide la radiación solar en la determinación đe las estaciones. Sin embargo, al aplicar su saber sobre este tema, mostraron frecuentes imprecisiones y un alto grado de confusión: ues verano porque el Sol está inclinado, e invierno porque está más recto» (chica de 15 años); «al estar la Tierra más lejos del Sol, la luz llega con un ángulo mayor, y por eso es verano» (chico de 14 años); «porque el plano donde está el Sol respecto al de la Tierra es inferior» (alumna de magisterio de 21 años).

\section{Implicaciones didácticas}

No es fácil relacionar la inclinación del eje đe rotación de la Tierra con la cantidad de radiación recibida por unidad de superficie. La comprensión del modelo puede facilitarse con el uso de simulaciones, por ejemplo, calcular las áreas iluminadas de superficies planas (una carpeta, un trozo de madera...) que se inclinan, y diseñar un modelo sencillo Tierra-Sol con un balón y un foco de luz (de Manuel y Montero 1992, Albaladejo et al. 1993). Este tipo de actividad puede ser eficaz, pero también puede generar nuevas representaciones erróneas si el alumno aplica literalmente algunos aspectos de la simulación a la realidad (por ejemplo, la escala).

Los resultados del anàlisis comparativo, lievado a cabo entre los cursos que habían realizado una actividad de simulación con otros que no lo habian hecho (Fig. 7), deben considerarse con cautela. La heterogeneidad de la muestra, así como otras variables (tipo de simulación realizada, ejemplos de aplicación usados en la actividad, el hecho de que ésta fue realizada por profesores diferentes, etc.) deben tenerse en cuenta en la interpretación. Los resultados más correctos no se observaron en cursos que habían realizado hacía un año la simulación, sino con los mayores, que la habían realizado tres cursos atrás; quizá al tener más información (formal o informal) habían sido capaces de aprender más significativamente el modelo. Sin embargo, algunas representaciones alternativas siguieron presentándose con firmeza en todos los cursos a pesar de haber realizado la simulación, especialmente al atribuir los veranos e inviernos a la distancia al Sol y a la rotación. Nusbaum y Novak (1976) y Nussbaum y Sharoni-Dagan (1983) investigaron cómo cambiaba la noción de la Tierra como cuerpo cósmico con una determinada estrategia didáctica, conciuyendo que buena parte de Ias concepciones subsisten a pesar de la instrucción.

Osborne (1991) sugiere la simulación escénica del modelo Sol-Tierra-Luna, con estudiantes que hacen los roles de la Luna, la Tierra y deI Sol. El mismo autor propone, para comprender las estaciones, diseñar situaciones problema y ofrecer a los alumnos dos explicacio- nes de por qué hay verano e invierno (la de la distancia al sol, y Ia correcta), y haciendo que éstos encuentren evidencias que estén de acuerdo con cada una de las hipótesis. En cualquier caso, conviene causar conflictos que cuestionen las concepciones de Ios estudiantes. En las entrevistas, por ejemplo, chicos y chicas convencidos que la rotación terrestre era la causa de los inviernos y veranos, veían su teoría «desvanecerse» al pedirles la causa de los días y las noches.

Nussbaum (1979) considera erróneo tratar aspectos conceptuales avanzados, dejando de lado, o dando por sabidas, Ia identificación y la caracterización de la propia Tierra como cuerpo cósmico. En este sentido, la propuesta de realizar simulaciones del modelo Sol-Tierra como actividades complementarias (aunque no únicas) debería ser posterior al aprendizaje de conceptos referentes a Ia Tierra como cuerpo en el espacio (tamaño, forma, inclinación del eje, representación espacial, etc.).

El lenguaje y los textos utilizados en clase de ciencias pueden inducir a ideas alternativas y confusiones. Que los estudiantes memoricen ( $i$ aprendan?) fragmentos de textos o expresiones como los extremos de la perpendicular a la linea de los equinoccios se llaman solsticios [... [ En la actualidad el soslticio de verano corresponde en el hemisferio sur al perihelio, mientras que en el hemisferio norte, al afelio" (extraído literaimente de un libro de texto dirigido a estudiantes de 14 a 15 años) resulta poco adecuado para que comprendan el modelo Sol-Tierra y lo relacionen con las estaciones. La instrucción debe favorecer aprendizajes significativos, y el lenguaje usado en clase debe estar más de acuerdo con la posibilidad de comunicar ideas que con el conocimiento de los términos concretos que usan los científicos (Izquierdo 1993). El lenguaje científico interacciona con el significado del lenguaje cotidiano (Llorens et al. 1989). Términos bastante comunes como rotación o hemisferio se confunden con otros conceptos o se aplican incorrectamente. Así, en las entrevistas se constató que algunos alumnos, y no solamente entre los más jóvenes, utilizaron el término rotación refiriéndose en realidad al movimiento de traslación. Otros al hablar de los hemisferios estaban refïriéndose en realidad a las mitades que resultaban de partir la Tierra por un plano que pasase por Ios polos (Fig. 6,9). Ello debe tenerse en consideración en la interpretación de algunos porcentajes dados en la tabla II. Menos grave es asignar equivocadamente conceptos como fuerza (los rayos solares en verano llegan con mucha más fuerza que en invierno) atribuida a los rayos del Sol.

Conviene que en el currículo de la enseñanza secundaria obligatoria se aborden contenidos de astronomía, especialmente del sistema Sol - Tierra - Luna. Los líbros de texto o los materiales curriculares deben incluir estos contenidos, y las clases de ciencias no pueden obviarlos. Nuestros alumnos y alumnas tendrán estaciones cada año de su vida, notarán que el período de luz de los días varía a lo largo del año que muchas noches verán la Luna de diferente forma y posiblemente hayan visto o verán algún eclipse. A partir de estos hechos y fenómenos que forman parte de la vida cotidiana, Ios chicos y chicas 
construyen representaciones y pueden elaborar sus teorías para explicar cada uno de ellos. Pero, como aquí se ha constatado, es muy común que las ideas de la mayor parte del alumnado sobre alguno de estos fenómenos, sean erróneas o muestren un alto grado de confusión, incluso en los últimos cursos de bachillerato y en buen número de futuros maestros y maestras.

\section{AGRADECIMIENTOS}

Agradezco a todos aquellos profesores y profesoras que me permitieron desinteresadamente realizar esta investigación con sus estudiantes. Y por supuesto mi gratitud para con estos áltimos, sin los cuales nada hubiera sido posible. Debo también agradecer a Neus Sanmartí sus orientaciones iniciales en el diseño experimental y en la interpretación de los resultados. También quisteta expresar mi agradecimiento a Teresa Correig y a Joan Bach, cuyas sugerencias mejoraron un primer borrador.

\section{NOTA}

Este trabajo forma parte de un proyecto de investigación realizado gracias a una licencia de estudios concedida por el Departament d'Ensenyament de la Generalitat de Catalunya (DOGC 1602, 3/6/92).

\section{REFERENCLAS BIBLIOGRÁFICAS}

ALBALADEJO, C., GRAU, R, GUASCH, E., DE MANUEL, J., 1993. La Ciència a l'Aula. Activitats d'aprenentatge en Ciències Naturals. (Barcanova. Colección Educación: Barcelona).

CAMINO, N., 1995. Ideas previas y cambio conceptual en astronomía. Un estudio con maestros de primaria sobre el día y la noche, las estaciones y las fases de la Luna, Enseñanza de las Ciencias, 13(1), pp. 81-96

CLAXTON, G., 1984. Live and Learn. An introduction to the psychology of growth and change in everyday life. (Harper \& Row: Londres). (Trad. cast. 1987, Vivir y aprender, Alianza: Madrid)

DEMANUEL, J. y MONTERO, A.M., 1992. Els Jocs Olímpics, una font de recursos per a la didàctica de les Ciències Naturals. De la Primària al Batxillerat, Guix, 173, pp. 7-13.

GIORDAN, A., DE VECCHI, G., 1987. Les origines du savoir (des conceptions des apprenants aux concepts scientifiques). (Delachaux \& Niestlế SA: París). (Trad. cast. 1988, Los origenes del saber. De las concepciones personales a los conceptos científicos, Díada editores: Sevilla).

IZQUIERDO, $M_{.,}$1993. El llenguatge científic a secundària. Guix, 183, pp. 23-30.

IONES, B.L., LYNCH, P.P., REESINK, C., 1987. Children's conceptions of the earth, sun and moon, Int. J. Sci. Educ., 9 (1), pp. 43-53.

KAPTERER, J.N., DUBOIS, B., 1981. Échec à la science. (NER: París).

KLEIN, C.A., 1982. Children's concepts of the Earth and Sun: a cross-cultural study, Science Education, 65(1), pp.95-107.

LLORENS, J.A., DE JAIME, M.C., LLOPIS, R., 1989. La función del ienguaje en un enfoque constructivista del aprendizaje de las ciencias, Enseñanza de las Ciencias, 7(2), pp. 111-119.
NUSSBAUM, J., 1979. Children conceptions of the earth as a cosmic body, Science Education, 65 (1), pp. 83-93.

NUSSBAUM, J., NOVAK, J.D., 1976. An assesment of children's concepts of the Earth utilizing structured interviews, Science Education, 60(4), pp. 535-550.

NUSSBAUM, J., SHARONI-DAGAN, N., 1983. Changes in second grade children's preconceptions about the earth as a cosmic body resulting from a short series of audio-tutorial lessons, Science Education, 67(1), pp. 99-114.

OSBORNE, J., 1991. Approaches to the teaching of AT16- the Earth in space: issues, problems and resources, School Science Review, 72 (260), pp. 7-15.

OSBORNE, R, FREYBERG, P., 1985. Learning in Science. The implications of Children's science. (Heinemann Publishers). (Trad. cast., 1991, Elaprendizaje de las ciencias. Implicaciones en la ciencia de los alumnos, Narcea ediciones: Madrid).

OSBORNE, R., WIT TROCK, M., 1983. Learning science: a generative process, Science Education, 67, pp. 591-599.

POSNER, G.J., STRIKE, K.A, HEWSON, P.W. , GERTZOG, W.A. 1982. Accommodation of scientific conception: Toward a theory of conceptual change, Science Education, 66, pp. $211-227$.

POZO, J.1., 1989. Teorías cognitivas del aprendizaje. (Morata: Madrid).

POZO, J.I., SANZ, A., GÓMEZ CRESPO, M.A., LIMÓN, M., 1991. Las ideas de ios alumnos sobre la ciencia: una interpretación desde la psicología cognitiva, Enseñanza de las Ciencias, 9, pp. 83-94.

SCHOON, K.J., 1992. Students'alternative conceptions of Earth and Space, Journal of Geological Education, 40, pp. 209-214. 